statistics. In general, the use of summary statistics obtained from other statistical packages would seem preferable except where the sample sizes are small and the calculation is a “one-off'. There are no facilities in the program for inputting data from other computer software or for saving any raw data input. Thus a rerun with an additional sample, for example, would necessitate repunching all the data. The only facilities for printing entered data and results is by using the "print-screen" key.

For use in conjunction with Statistics with Confidence and for calculating confidence intervals, CIA is a handy program, but for medical reseach projects using reasonable quantities of data it inevitably must supplement a more comprehensive computer statistical package.

LINDA M ANDERSON

Anaerobic Infections in Humans. Ed SM Finegold, W Lance George. (Pp 851; \$149.) Academic Press Inc. 1989. ISBN 0-12256745-5.

There are few authoritative texts on anerobic infections. In 39 chapters, this book covers, virtually all aspects of the subject, from the historical aspects, through classification, laboratory diagnosis, sensitivity testing, descriptive chapters on different system infections, to therapeutic aspects, with additional chapters on such aspects as pathogenicity factors, host-defence mechanisms and the role of anaerobes as normal flora. The chapters are, in general, detailed and well written but the style varies from chapter to chapter. Some chapters have helpful summaries; others do not. The reference style, too, is inconsistent.

Much of the information, references, and techniques in this book is derived from American sources and based on American practice. For example, metronidazole is mentioned only briefly (three page references in the Index) whereas clindamycin and cefoxitin receive extensive coverage ( 27 and 11 references, respectively).

These are perhaps small criticisms. This is an extensive, well illustrated, and valuable reference source for all those interested in anaerobic infections.

B WATT

HIV Detection by Genetic Engineering Methods. Ed PA Luciw and KS Steimer. (Pp 312; \$119.50.) Marcel Dekker Inc. 1989. ISBN 0-8427-7900-2.

This book comprises 15 chapters written by a variety of authors from HIV research in both the academic and commercial based sectors of the USA. The scene is set by the first two chapters which give succinct accounts of the molecular biology and epidemiology of HIV infection and AIDS. In the following seven chapters the use of recombinant proteins and synthetic peptides as reagents for immunoblot and enzyme immunoassays to detect and characterise the antibody response to HIV infection are detailed. Emphasis is then switched to antigen and nucleic acid detection techniques in serum, infected cells, and tissues. The chapters include much of the practical detail needed to get these techniques working, and examples of results are shown which highlight the importance of these methodologies. The final chapter is not about HIV but briefly reviews HTLV1 and
HTLV2 viruses, perhaps misplaced in a book with this title.

Overall, the book is well illustrated and clearly written, with the chapters individually referenced, making it recommended reading for all individuals who are involved in the detection of HIV for diagnosis or research purposes.

P GRINT

Chromosomal Variation in Man. A Catalog of Chromosomal Variants and Anomalies. 5th ed. DS Borgaonkar. (Pp 803; £85) Alan R Liss Inc. 1989. ISBN 08451-4275-5.

This is the fifth edition of Chromosome Variation in Man and like its predecessors is a most welcome addition to the literature of clinical cytogenetics. The book is divided into 11 sections which clearly and concisely cover the major categories of chromosome anomalies in man. The principal part is devoted to structural abnormalities of human chromosomes. The book stands or falls on this section (which comprises three quarters of the entries) and by and large it is covered admirably. The listing is comprehensive, up to date, and there is a short summary of the more interesting cases. Many new microdeletion syndromes such as the proximal $17 p$ deletion in Smith-Magenis syndrome and the association of proximal 15q deletions with both Prader Willi syndrome and Angelman's syndrome, have already been listed. One small criticism concerns the dearth of entries for fragile $\mathrm{x}$ syndrome. This condition provides numerous problems for diagnostic laboratories and would certainly justify a larger number of entries.

The remaining sections of the book complete an excellent reference work that is thoughtfully presented, comprehensive, ye up to date. The book is not cheap but should prove invaluable to anyone working in clinical cytogenetics whether in a research environment or busy diagnostic laboratory. AM POTTER

Viral and Autoimmune Hepatitis. HP Dienes. (Pp 107; 54 fig. soft cover DM 98.) Gustav Fischer Verlag. 1989. ISBN 0-89574280-2.

It is the custom in many continenta European countries for theses to be printed and bound, and a few are published commercially. This, I suspect, is the history of this slim paperback for the text has the conventional major subdivisions into introduction, materials and methods, results, and discussion. Potential readers however, should not be misled into thinking that this is a relatively trivial publication: it is not. The author has accumulated a wealth of interesting observations on liver biopsy specimens from patients with hepatitis $B$, non-A non-B hepatitis, autoimmune hepatitis, and on liver samples from animals with HSV hepatitis. The book is beautifully illustrated with light micrographs, all in colour, and electron micrographs.

This book is not, I advise, for the general reader in histopathology. It is a specialist text which many liver pathologists will read with interest.

JCE UNDERWOOD
NOTICES

\begin{tabular}{|l|}
\hline \multicolumn{1}{|c|}{ First ICR } \\
International Clinical Symposium \\
"The Role of Clinical Measurement in the \\
Evaluation of New Drugs" \\
Sheraton Hotel: Edinburgh: 8-10 May \\
1990 \\
Organised by Inveresk Clinical Research \\
Further details from: \\
CEP Consultants Ltd, \\
26-28 Albany Street, \\
Edinburgh EH1 3QH, UK \\
(Tel: 031 557 2478; \\
Telefax: 031 557 5749)
\end{tabular}

\section{Second International Conference} on Small Cell Lung Cancer

May 11-12 1990

Milano Marittima, Ravenna, Italy

Organised by Augustea SRL

Further details from:

Dr Nadia Colaiuda

Augustea SRI

Via di Roma 86

48100 Ravenna, Italy

Tel: (0544 30329-30068)

Fax: (0544 34064)

\section{Association of Clinical Pathologists}

\section{JUNIOR MEMBERSHIP}

Junior Membership of the Association of Clinical Pathologists is available to trainees in all branches of pathology for up to six years or until they attain consultant status. The annual subscription is $£ 18$, which may be claimed against tax.

All Junior Members receive monthly copies of the Journal of Clinical Pathology. Other benefits include membership of the Junior Members' Group and a regular junior members' newsletter, the ACP Newsletter, and all the documents regularly sent to full members of the Association. These include the twice yearly summary of pathology courses included in the ACP Postgraduate Education Programme.

For Junior Membership apply to: Dr JD Davies, Education Secretary, University Department of Pathology, Bristol Royal Infirmary, Bristol BS2 8HW.

\section{ACP Locum Bureau}

The Association of Clinical Pathologists runs a locum bureau for consultant pathologists.

Applicants with the MRC Path who would like to do locums and anyone requiring a locum should contact $\mathrm{Dr}$ David Melcher, Histopathology Department, Sussex County Hospital, Eastern Road, Brighton BN2 5BE. 